Original Research

\title{
Limb Dominance Does Not Affect Y-Balance Test Performance in Non-Athlete Adolescents
}

\author{
Carissa A. Stoddard, PT, DPT, PCS ${ }^{1}$, Sharon Wang-Price, PT, PhD, OCS, FAAOMPT ${ }^{1}$, Satoko E. Lam, PT, MSPT, CKTP² \\ ${ }^{1}$ School of Physical Therapy, Texas Woman's University, ${ }^{2}$ Rehab \& Therapy Services, Children's Health \\ Keywords: reliability, single leg stance, reach, postural stability, dynamic balance \\ https://doi.org/10.26603/001c.30996
}

International Journal of Sports Physical Therapy

Vol. 17, Issue 2, 2022

\section{Background}

The Lower Quarter Y-Balance Test (YBT-LQ) has been shown to be reliable for assessing dynamic balance in children and adolescents. However, limited research is available about the effects of leg dominance on YBT-LQ performance in adolescents. In addition, there is no consensus on the use of maximum reach or mean reach distance being a better measure of YBT-LQ performance.

\section{Hypothesis/Purpose}

The purposes of this study were to determine if there is a difference in the YBT-LQ performance between the dominant and non-dominant limbs in non-athlete adolescents, and to compare the reliability of the maximum reach scores to that of the mean reach scores in this population.

\section{Study Design}

Prospective cohort study

\section{Methods}

Twenty-six healthy non-athlete adolescents (13.6 \pm 1.0 years, 22 girls, 4 boys) performed the YBT-LQ on two separate days while the same investigator scored their performance. Paired $t$-tests were used to compare reach distances on dominant and non-dominate stance limbs. Intraclass correlation coefficients $\left(\mathrm{ICC}_{3,1}\right)$ were calculated for the maximum and mean reach distances for three directions (anterior, posterolateral, posteromedial) and the composite scores on each limb.

\section{Results}

There was no significant difference in YBT-LQ performance between dominant and non-dominant stance limbs $(p>0.05)$. Overall, the between-day intra-rater reliability for maximum reach and mean reach scores was moderate-to-good for both limbs $\left(\right.$ ICC $_{3,1}=$ $0.59-0.83)$, but was poor for the composite score on the dominant limb $\left(\operatorname{ICC}_{3,1}=0.42\right.$ ) and maximum anterior reach on non-dominant limb $\left(\mathrm{ICC}_{3,1}=0.48\right)$.

\section{Conclusion}

Limb dominance does not seem to be a factor for YBT-LQ performance in this population. The YBT-LQ appears to be a reliable tool for dynamic balance assessment in non-athlete adolescents using the individual score of each direction. The use of mean reach measures seems to slightly improve reliability, specifically the anterior reach direction, in this

\footnotetext{
Corresponding Author:

Carissa Stoddard, PT, DPT, PCS

School of Physical Therapy

Texas Woman's University

5500 Southwestern Medical Ave

Dallas, TX 75235

512-965-0740

castoddard1@gmail.com
} 
population.

\section{Level of Evidence}

Level $2 b$

\section{INTRODUCTION}

Postural stability is considered to be an important indicator of neuromusculoskeletal health. ${ }^{1}$ Specifically, postural stability in children and adolescents is influenced by physiological functions such as muscular strength and neuromuscular development in childhood. ${ }^{2}$ Dynamic postural control is an important prerequisite to the development of fundamental movement skills and activities of daily living in children ${ }^{3,4}$ and is usually established in the first decade of life, whereas proficiency is acquired as children age, develop, and interact with their environment. ${ }^{4}$ Without mastery of balance abilities in the early years of childhood, children's performance in more complex movements associated with development and sports may be diminished, and they may be at a higher risk for injury during activity participation as adolescents or adults. ${ }^{4}$ It is known that decreased balance and dynamic postural control are associated with lower extremity injuries among adolescent athletes ${ }^{5,6}$ and adult athletes, ${ }^{7-10}$ and that adolescents have a higher risk of sport-related injury than younger children. ${ }^{5}$ Therefore, assessment of dynamic postural stability and balance is important in identifying musculoskeletal impairments, risk for injury, ${ }^{5-10}$ and monitoring recovery from injury. ${ }^{11-15}$

Tests such as the Lower Quarter Y-Balance Test (YBTLQ) are often used to predict injuries in high school and college athletes. ${ }^{7,8,10}$ They are also used to assess dynamic balance and postural control in various populations, including healthy, recreationally active adults, ${ }^{16-18}$ college and professional athletes, $7,9-11,19-22$ and adults recovering from injury. ${ }^{12,15,23-25}$ The YBT-LQ is an instrumented tool that was developed using components of the Star Excursion Balance test to standardize performance of dynamic balance and postural stability. ${ }^{11}$ To perform the YBT-LQ, a participant must maintain single limb stance on a stationary platform while pushing a movable target with the opposite foot in the anterior (ANT), posterior-medial (PM) and posteriorlateral (PL) reach directions (Figure 1).

The reliability of the YBT-LQ has been extensively researched in populations of various ages. For adult populations, the YBT-LQ was found to have good-to-excellent inter-rater reliability $\left(\mathrm{ICC}_{2,1}=0.80-1.00\right)$ and intra-rater reliability $\left(\mathrm{ICC}_{3,1}=0.85-0.91\right) .{ }^{11,26}$ For adolescents, the within-day inter-rater reliability was reported to be excellent $\left(\mathrm{ICC}_{2,1}=0.96-0.99\right),{ }^{27}$ whereas the within-day and between-day intra-rater reliability was found only to be fairto-good $\left(\mathrm{ICC}_{3,1}=0.57-0.91\right) .{ }^{27-29}$ Lastly, for pre-adolescent children, both the within-day $\left(\mathrm{ICC}_{2,1}>0.995\right)$ and betweenday $\left(\mathrm{ICC}_{2,1}=0.91-0.97\right.$ ) inter-rater reliability were excellent. The between-day intra-rater reliability was less than excellent, but the ICC values $(0.71-0.84)$ still showed moderate-to-good reliability. ${ }^{3}$ In addition, although many studies $3,11,27,28$ reported reliability outcomes using maximum reach distances for data analysis, other studies ${ }^{26,29}$ suggested using mean reach for YBT-LQ performance analysis. No consensus has been reached regarding which measure is

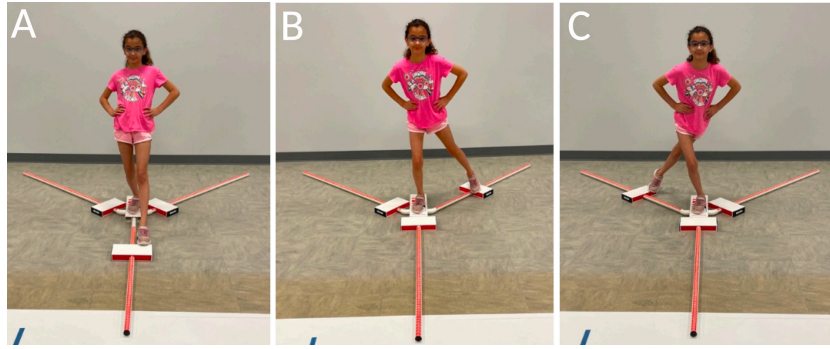

Figure 1. Performance of Y-Balance Test - Lower Quadrant: (A) right anterior reach, (B) right posteromedial reach, $(\mathrm{C})$ right posterolateral reach

superior, specifically among adolescent populations.

Leg dominance has been reported to be a risk factor associated with non-contact lower extremity injury among athletes. ${ }^{30,31}$ Clinicians often compare performance outcomes on functional balance tests between limbs to determine risk of injury, to evaluate presence of instabilities, to assess progress, or to consider readiness for return-to-sport activities. However, this assumes that both limbs are functionally equal and symmetrical prior to injury. ${ }^{32-34}$ Hoffman et al. ${ }^{33}$ measured center of pressure excursion (measure of dynamic balance) while a group of healthy adults were performing a static unipedal stance on a force platform. They found no significant differences between dominant and non-dominant limbs. Similarly, two other studies 32,34 found symmetry between dominant and non-dominant limbs in healthy adults during a single limb stance on a movable platform. However, Promsri et al. ${ }^{30,35}$ reported significant differences in postural control when investigating single leg stance on a firm surface and on a multiaxial unstable board between preferred and non-preferred limbs of healthy adults.

Furthermore, literature suggests no effects of leg dominance on a single limb stance in adolescent and teen populations. Mala et al. ${ }^{36}$ and Bigoni et al. ${ }^{37}$ found that limb preference had no significant effect on postural stability during a static single leg stance on a pressure mat among elite teenage soccer players and in pre-pubescent male soccer players, respectively. To date, few studies have used the YBT-LQ to compare limb differences in young adolescent populations. Muehlbauer et al. ${ }^{38}$ assessed dynamic balance in young soccer players using the YBT-LQ. Similar to the results of the Mala et al. and Bigoni et al. studies, Muehlbauer et al. ${ }^{38}$ did not find differences between dominant and nondominant limbs. In contrast, Breen et al. assessed performance of the YBT-LQ in children and adolescent athletes and found a significant asymmetry between limbs in the posterior-medial and posterior-lateral reach directions among children aged 10-12 years compared to teens aged 16-18 years, although limb dominance was not reported. 39 The populations of the above-mentioned studies were subelite or elite athletes, thus limiting the generalizability of 
results to other populations. To date, no other studies have examined effects of leg dominance on postural stability in untrained or non-athletic adolescents. Specifically, it is not clear if leg dominance would affect YBT-LQ performance in this population.

Young adolescents are at higher risk for lower extremity injury compared to older teens and adults, ${ }^{4,27,39-41}$ in part due to the rapid rate of growth occurring during adolescence, ${ }^{2,40,42}$ which could affect dynamic balance significantly. As competitive athletes were the populations of interest in the previous studies that examined leg dominance effects on dynamic balance, the target participants in this study were adolescents with a variety of body types and those who did not participate in organized or elite sport training. In addition, as discussed earlier, it is difficult to compare the YBT-LQ study results because both maximum and mean reach distances were used interchangeably. Therefore, the primary purpose of this study was to determine whether or not the YBT-LQ scores would be different between the dominant and non-dominant limbs in non-athlete adolescents aged 12-16 years. The secondary purpose of this study was to compare the between-day intra-rater reliability of the maximum reach performance to that of mean reach performance on YBT-LQ in non-athlete adolescents. The reliability using these two outcome measures was anticipated to be in agreement with other studies performed on this age group. ${ }^{27-29}$

\section{METHODS}

\section{SUBJECTS}

Using $\mathrm{G}^{*}$ Power version $3.1,{ }^{43}$ an a priori power analysis was performed to calculate the sample size needed to detect a significant difference between dominant and non-dominant limbs. A total of 26 participants was required to achieve a power of 0.80 using an $\alpha$ of 0.05 and a medium effect size of 0.50 . The effect size of 0.50 was chosen based on the limb difference findings of a previous YBT-LQ study in healthy adults. 30

The study was approved by the Institutional Review Board at Texas Woman's University. Healthy adolescents aged 12-16 years who did not actively participate in organized sports or in elite sport training were recruited for participation in the study from a local junior/senior high school. Eligible participants were excluded from the study if they reported: lower extremity injury or surgery within the prior six months, current or recent vestibular disorder within the prior three months, currently being treated for inner ear, sinus, upper respiratory infection or head cold, concussion within the prior three months, pregnancy, or musculoskeletal or neuromuscular pathology/diseases that could affect dynamic balance. Once the participant was determined to be eligible for the study, the participants and their parent or legal guardian read and signed the informed consent form.

\section{INSTRUMENT}

The Y-Balance Test $\mathrm{Kit}^{\mathrm{TM}}$ was used to assess dynamic balance in this study. The kit consists of a stance platform to

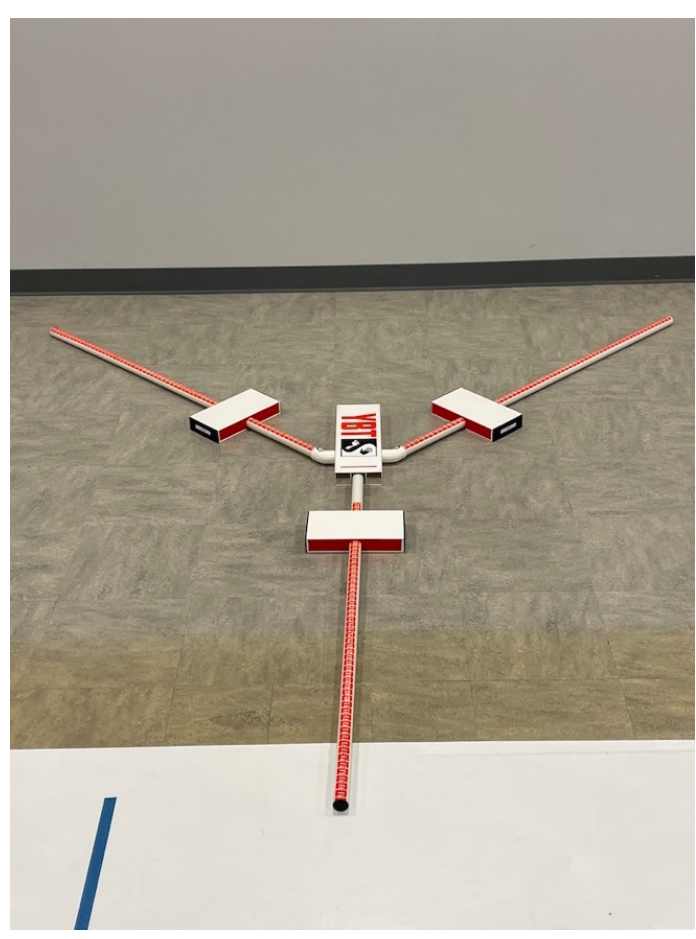

Figure 2. Y-Balance Test Kit

which three pieces of PVC pipe are attached in the ANT, PM, and PL directions. ${ }^{11}$ The posterior pipes are positioned 135 degrees from the anterior pipe, with 90 degrees between the posterior pipes. Each pipe is marked in 5-millimeter increments for measurement. A reach indicator slides on each pipe so that the participant pushes it with one limb while maintaining a single leg stance on the center stance platform (Figure 2). The distance that the indicator traveled was recorded as reach distance.

\section{PROCEDURES}

Prior to the YBT-LQ testing, participants completed an intake form and self-reported their height, weight, and dominant limb. Dominant limb was defined as the preferred leg used to kick a ball. ${ }^{32,34,44}$ Leg length was measured for each lower extremity on each participant while lying in the supine position with hips and knees extended. The investigator measured the distance from the most inferior aspect of the anterior superior iliac spine to the most distal portion of the medial malleolus with a tape measure. Leg length was used to normalize the reach distances collected during the YBT-LQ testing.

The YBT-LQ testing protocol was performed as described by Plisky and Gorman. ${ }^{11}$ All participants were instructed with the YBT-LQ test and given a demonstration of proper performance of the YBT-LQ by the by a single rater (i.e., principal investigator). Each participant stood on one leg in the center of the stance platform with the most distal aspect of their athletic shoe at the starting line. While maintaining a single-leg stance, the participant was asked to push the reach indicator along the pipe with the free limb in the ANT, $\mathrm{PM}$, and PL directions in relation to the stance foot. Each participant was allowed up to six practice trials on each leg 
Table 1. Demographic Characteristics of the Participants, presented as mean (SD) or count.

\begin{tabular}{lcc}
\hline & Session $\mathbf{1}(\mathbf{n}=\mathbf{2 6})$ & Session $2(\mathbf{n}=24)$ \\
\hline Age $($ years $)$ & $13.62(0.98)$ & $13.63(1.01)$ \\
Height $(\mathrm{cm})$ & $156.94(9.05)$ & $156.58(9.16)$ \\
Weight $(\mathrm{kg})$ & $59.75(11.80)$ & $59.23(10.55)$ \\
BMI $\left(\mathbf{k g} / \mathrm{m}^{2}\right)$ & $23.38(4.1)$ & $24.23(4.35)$ \\
Leg Length $(\mathrm{cm})$ & $85.65(5.5)$ & $85.52(5.6)$ \\
Leg dominance & Right $=22$ & Right $=21$ \\
& Left $=4$ & Left $=3$ \\
\hline
\end{tabular}

in each reach direction prior to formal testing. ${ }^{11,29}$ Testing occurred within 20 minutes after completion of practice trials. ${ }^{11}$ Each participant performed three reach trials ${ }^{29}$ in each direction for both limbs in the standardized testing order described by Plisky and Gorman. ${ }^{11}$ Each participant started in the ANT direction with the left foot while standing on the right leg, followed by standing on the left leg and reaching in the ANT direction with the right foot. This procedure was repeated for the PM reach direction followed by the PL reach directions. ${ }^{11}$ Additional testing trials were added if the first three trials were deemed unsuccessful. Unsuccessful trials were discarded and repeated if the participant 1) failed to maintain unilateral stance on the platform, 2) failed to maintain the reach foot contact with the reach indicator in the target direction while in motion, 3) used the reach indicator for stance support, or 4) failed to return the reach foot to the starting position under control. The starting position for the reach foot is defined by the area immediately between the standing platform and the pipe opposite the stance foot. ${ }^{11}$ If a successful testing trial was not completed within three reaches, additional trials were performed up to six reaches in a single direction until a successful trial was completed. If a participant was unable to perform a successful trial in six attempts, the participant failed that direction.

Testing was administered and scored by the principal investigator, a licensed physical therapist with greater than two years' experience in YBT-LQ, who repeated the YBT-LQ a second time the next day on the same participants in order to determine between-day intra-rater reliability. Although the same investigator scored the YBT-LQ both times, each participant's scores of the first YBT-LQ were not available to the testing investigator during the second testing. The greatest successful reach distance from three trials in a single direction was used as maximum reach. Mean reach was also calculated for each direction by averaging the reach distances of three trials: [(Reach 1 + Reach 2 + Reach 3) / 3]. Maximum reach and mean reach distance in each direction were normalized as a percentage of leg length to allow for comparison across participants in this and other studies. The following formula was used to calculate normalized reach score: [(maximum or mean reach/limb length) x 100]. The maximum reach or mean reach for all three directions was summed and used as a composite reach score (COMP) for analysis of overall performance on the test. The following formula was used to calculate composite score: [(ANT reach distance + PM reach distance + PL reach distance)/ (3 $\mathrm{x}$ limb length)] $\mathrm{x} 100$.

\section{DATA ANALYSIS}

Means and standard deviations were calculated for participants' demographics, as well as the reach distances of all three directions and composite score for each limb. Paired $t$-tests were used to compare the reach distances in all three directions and the composite scores between the dominant and non-dominant limbs. Intraclass correlation coefficients $\left(\mathrm{ICC}_{3,1}\right)$ were calculated to determine the between-day intra-rater reliability with a $95 \%$ confidence interval (CI). Interpretation of ICC values were as follows: ICC < 0.50 : poor reliability, ICC $=0.50-0.74$ : moderate or fair reliability, ICC $=0.75-0.89$ : good reliability, and ICC > 0.90: excellent reliability. ${ }^{45}$ Standard error of measurements (SEMs) were calculated to estimate the amount of error using the formula $\mathrm{SEM}=\mathrm{SD}^{*} \sqrt{(1-I C C)} \cdot{ }^{28}$ Minimal detectable changes (MDCs) also were computed for clinical interpretation using the equation: $\mathrm{MDC}_{95 \%}=\mathrm{SEM} * 1.96 * \sqrt{2} .{ }^{28}$ All statistical analyses were completed using IBM SPSS statistics for Macintosh, version 25 (IBM Corp., Armonk, NY), and the $\alpha$ level was set at 0.05 for all statistical analyses.

\section{RESULTS}

Twenty-six healthy adolescents ( 22 girls, 4 boys) with an average age of $13.6 \pm 1.0$ years were enrolled in the study. Two participants (girls) did not return for the second day testing, resulting in data analysis of 26 subjects for comparisons between dominant and non-dominant stance limbs and 24 subjects for the between-day intra-rater reliability. Demographic data of all participants is presented in Table 1.

Table 2 presents the maximum and mean reach distances from the data collected from 26 participants during Session 1 , corresponding normalized values in all three directions, and the composite scores for the dominant and non-dominant limb. Two participants (girls) were not able to complete any ANT reach for both limbs, two additional participants (girls) only completed the ANT reach on the dominant limb, two different participants (a girl and a boy) only completed the ANT reach on the non-dominant limb, and one participant (girl) only completed a single trial on the dominant limb. These participants were unsuccessful at completing the ANT reach because they failed to main- 
Table 2. The Maximum and Mean Reach Distances of the Y-Balance Test-Lower Quarter (YBT-LQ) presented as mean $(\mathrm{SD})(\mathrm{n}=26)$

\begin{tabular}{ccccc}
\hline & $\begin{array}{c}\text { Maximum Reach } \\
\text { Distance }(\mathrm{cm})\end{array}$ & $\begin{array}{c}\text { Normalized Maximum Reach } \\
\text { Distance }(\%)\end{array}$ & $\begin{array}{c}\text { Mean Reach } \\
\text { Distance }(\mathrm{cm})\end{array}$ & $\begin{array}{c}\text { Normalized Mean Reach } \\
\text { Distance (\%) }\end{array}$ \\
\hline Dominant Limb & & & & \\
ANT* & $45.35(20.53)$ & $53.27(24.24)$ & $43.88(19.92)$ & $51.56(23.53)$ \\
PM & $85.08(11.89)$ & $99.44(13.26)$ & $82.79(11.27)$ & $96.77(12.58)$ \\
PL & $79.81(20.68)$ & $93.34(24.28)$ & $76.09(20.50)$ & $88.99(24.21)$ \\
COMP & $82.01(15.08)$ & $94.16(15.97)$ & $79.11(14.70)$ & $79.11(14.70)$ \\
Non-Dominant Limb & & & $49.68(23.0)$ \\
ANT & $44.54(20.82)$ & $51.95(24.30)$ & $42.59(19.7)$ & $98.48(14.97)$ \\
PM & $85.96(12.21)$ & $100.58(14.40)$ & $84.15(12.63)$ & $89.79(23.35)$ \\
PL & $79.08(20.34)$ & $92.50(23.99)$ & $76.76(19.83)$ & $79.32(12.81)$ \\
COMP & $81.68(13.00)$ & $92.54(16.51)$ & $79.32(12.81)$ & \\
\hline
\end{tabular}

ANT = anterior PM = posteromedial; $\mathrm{PL}=$ posterolateral; $\mathrm{COMP}=$ composite score.

$* \mathrm{n}=21 ;{ }^{\dagger} \mathrm{n}=22$

Table 3. Intraclass Correlation Coefficients (ICCs), Standard Error of Measurement (SEM), Minimal Detectable Change (MDC) of the YBT-LQ in Non-athlete Adolescents $(n=24)$

\begin{tabular}{ccccccc}
\hline \multicolumn{2}{c}{ Using Maximum Reach Distance } & \multicolumn{2}{c}{ Using Mean Reach Distance } \\
\hline & $\mathrm{ICC}_{3,1}(95 \% \mathrm{Cl})$ & $\mathrm{SEM}(\mathrm{cm})$ & $\mathrm{MDC}_{95 \%}(\mathrm{~cm})$ & $\mathrm{ICC}_{3,1}(95 \% \mathrm{Cl})$ & $\mathrm{SEM}^{(\mathrm{cm})}$ & $\mathrm{MDC}_{95 \%}(\mathrm{~cm})$ \\
\hline Dominant Limb & & & & & & \\
ANT $^{*}$ & $0.73(0.44-0.88)$ & 3.87 & 10.72 & $0.69(-0.38-0.86)$ & 4.19 & 11.62 \\
PM & $0.81(0.60-0.91)$ & 5.58 & 15.58 & $0.78(0.56-0.90)$ & 5.57 & 15.43 \\
PL & $0.74(0.47-0.86)$ & 6.82 & 18.91 & $0.70(0.41-0.86)$ & 7.83 & 21.69 \\
COMP & $0.42(0.03-0.70)$ & 9.13 & 25.30 & $0.43(0.05-0.71)$ & 8.84 & 24.51 \\
Non-Dominant Limb & & & & & \\
ANT ${ }^{\dagger}$ & $0.48(0.06-0.75)$ & 6.22 & 17.25 & $0.59(0.21-0.81)$ & 5.15 & 14.29 \\
PM & $0.70(0.42-0.86)$ & 7.73 & 21.44 & $0.69(0.41-0.86)$ & 7.75 & 21.48 \\
PL & $0.80(0.58-0.91)$ & 6.31 & 17.48 & $0.83(0.63-0.92)$ & 5.81 & 16.11 \\
COMP & $0.75(0.51-0.88)$ & 5.93 & 16.45 & $0.75(0.51-0.89)$ & 5.87 & 16.27 \\
\hline
\end{tabular}

$\mathrm{CI}=$ confidence interval; $\mathrm{ANT}=$ anterior; $\mathrm{PM}=$ posteromedial; $\mathrm{PL}=$ posterolateral; $\mathrm{COMP}=$ composite score.

$* \mathrm{n}=19 ; \dagger \mathrm{n}=20$

tain unilateral stance on the platform or failed to return the reach foot to the starting position under control. Consequently, ANT reach from 21 participants' dominant limbs and ANT reach from 22 participants' non-dominant limbs were included for data analysis. Paired $t$-tests showed no significant differences between the dominant and nondominant limbs in all reach directions ( $p=0.054-0.973)$.

Because two participants (girls) did not return for Session 2 and these two girls completed ANT reach on both limbs during Session 1, ANT reach from 19 participants' dominant limbs and ANT reach from 20 participants' nondominant limbs were included for the reliability analysis. The reliability results including ICCs, SEM, and MDCs are presented in Table 3. The ICCs showed the between-day intra-rater reliability of the YBT-LQ for maximum reach to be moderate-to-good in all reach distances on both limbs $\left(\mathrm{ICC}_{3,1}=0.70-0.81\right)$, except for the non-dominant ANT reach and dominant COMP score, which had poor reliability $\left(\mathrm{ICC}_{3,1}=0.42-0.48\right)$. The reliability for mean reach distances were found to be moderate-to-good in all directions on both limbs $\left(\mathrm{ICC}_{3,1}=0.59-0.83\right)$. The reliability was good using COMP scores of mean reach distances for non-dominant stance limb $\left(\mathrm{ICC}_{3,1}=0.75\right)$, but was poor for dominant stance limb $\left(\mathrm{ICC}_{3,1}=0.43\right)$.

\section{DISCUSSION}

The results of this study indicate that healthy, non-athlete adolescents had similar performance in the YBT-LQ either 
performing on the dominant limb or on the non-dominant limb. These results are in agreement with the previous studies regarding effects of limb dominance during balance and postural stability tasks among adult and athletic populations. It has been hypothesized that the use of modern training regimens focusing on bilateral exercises during sport is a possible reason for no significant differences being found between limbs during YBT-LQ performance among athletes. ${ }^{38}$ Subjects in the present study were identified to be recreationally active, so they may also participate in bilateral physical activities, although specifics were not tracked and these were not controlled for during analysis. There have also been reports that the YBT-LQ may not be sensitive enough to detect reach distance differences between limbs in young athletes. ${ }^{38,39}$

Further comparison of mean reach distances among adolescent participants in the present study showed normalized mean reach performance to be similar to that reported by Bulow et al. ${ }^{41}$ who demonstrated that physically active healthy adolescent females had mean reach distances from 57.0 (4.5) $\mathrm{cm}$ to $103.6(8.8) \mathrm{cm}$. However, Linek et al. ${ }^{29}$ reported higher normalized mean reach distances for a cohort of male adolescent football players from $67.7(8.6) \mathrm{cm}$ to $112.1(10.4) \mathrm{cm}$. Similarly, the normalized maximum reach distances reported by Muehlbauer et al. ${ }^{38}$ were higher for a cohort of young male adolescent soccer players, reporting $72.8(7.4) \mathrm{cm}$ to $121.8(12.1) \mathrm{cm}$, compared to the maximum reach distances of participants in this study. The differences among the studies may be due to different levels of training and physical activity between the study populations. Participants in this study were of similar adolescent age but were untrained and only recreationally active compared to sub-elite or elite athletes described by Linek at al. ${ }^{29}$ and Muehlbauer et al. ${ }^{38}$ Higher maximum reach distances reported among adult studies ${ }^{32-34}$ compared to those of the adolescents in this present study could be attributed to differences in age of subjects as this has been reported to have a significant effect on performance with older, more experienced individuals having a greater reach distance on the YBT-LQ. ${ }^{38}$

The results of this present study demonstrated moderate or good between-day reliability $\left(\mathrm{ICC}_{3,1}=0.70-0.81\right)$ for maximum reach in all three directions except the ANT reach on the non-dominant limb and the composite score on the dominant limb, which resulted in lower reliability values $\left(\mathrm{ICC}_{3,1}=0.42-0.48\right)$. These results are similar to other studies that investigated the between-day reliability of YBT-LQ in adolescent populations. ${ }^{27-29}$ Schwiertz et al. ${ }^{28}$ reported moderate-to-excellent reliability $\left(\mathrm{ICC}_{3,1}=0.69-0.96\right)$ for the PL and PM reach directions among healthy adolescents in sixth to tenth grade (equivalent to 11-16 years of age), and poor-to-fair reliability $\left(\mathrm{ICC}_{3,1}=0.40-0.69\right)$ for the ANT reach on the right leg among the seventh and ninth graders. However, Schwiertz et al. ${ }^{28}$ reported overall good reliability ICC $\left._{3,1}=0.83-0.96\right)$ for the composite score. Similarly, Greenberg et al. ${ }^{27}$ reported moderate-to-excellent reliability $\left(\mathrm{ICC}_{3,1}=0.68-0.91\right.$ ) for YBT-LQ in adolescent female athletes, whereas Linek et al. ${ }^{29}$ reported fair-to-excellent reliability $\left(\mathrm{ICC}_{3,1}=0.57-0.82\right)$ among male adolescent and teenage semi-professional athletes. ICC values of the current study were observed to be within the range of ICCs reported by Schwiertz et al. in the three individual directions, ${ }^{28}$ however, the reliability was slightly lower than that reported by Greenberg et al. ${ }^{27}$ and Linek et al. ${ }^{29}$ This could be in part due to the participants in the present study wearing shoes to perform the YBT-LQ, rather than barefoot testing, thus contributing to the slightly lower reliability values.

It is well documented in the literature that ANT reach distance is generally lower than reach distance in other directions and an ANT reach direction asymmetry between limbs on the YBT-LQ is associated with non-contact lower extremity injury prediction among adult athletes. ${ }^{7-10}$ It was observed that $23 \%(n=6)$ of the participants were unable to successfully complete the ANT reach trial on either their dominant or non-dominant limb, or both, and one additional participant completed only one successful trial on their non-dominant limb. The unsuccessful trials were primarily due to the participants failure to maintain unilateral stance on the platform or failure to return the reach foot to the starting position under control. In addition, $27 \%(n=7)$ of the participants had a between-limb ANT reach differences greater than the recommended cut-off $(>4 \mathrm{~cm})$ used to predict risk of non-contact lower extremity injury. ${ }^{7}$ Muehlbauer et al. ${ }^{38}$ also reported a significant ANT reach asymmetry between limbs in the elite male athletes of adolescent age. These findings suggest there may be significant variability of ANT reach performance among young adolescents, including ability to achieve a successful reach trial, irrespective of activity level or sport training. Further research is needed to identify contributing factors to ANT reach asymmetry in this age group. Therefore, caution should be taken when interpreting ANT reach results for the purposes of lower extremity injury prediction in adolescents.

Previous studies on reliability of YBT-LQ predominantly have used maximum reach distance to evaluate YBT-LQ performance, $3,11,27,28$ but two studies ${ }^{26,29}$ reported both maximum reach and the mean reach of the YBT-LQ performance. Schaffer et al. ${ }^{26}$ reported superior ICC, SEM, and MDC values with the mean reach. The present study also showed that the use of mean reach had improved reliability for the ANT and PL directions on the non-dominant limb and composite scores for both limbs. Although the ICC value of the ANT reach on the non-dominant limb was not high $\left(\mathrm{ICC}_{3,1}=0.59\right)$ when using mean reach distances, the reliability improved compared to the lower reliability $\left(\mathrm{ICC}_{3,1}=0.48\right)$ when maximum reach was used. Consequently, lower SEMs and smaller MDCs were found when mean reach was used for scoring. These findings reflect reports in previous literature ${ }^{26}$ with noted variability likely due to the effects of puberty and growth on adolescent performance compared to skeletally mature adults. Linek et al. $^{29}$ reasoned that fluctuating postures among adolescents resulted in less uniformity of results during individual attempts and reported this as a possible reason for larger deviations found in reliability of young male athletes compared to adult studies. Therefore, Linek et al. suggested that the average of three measurements be used for reliability in the adolescent population. ${ }^{29}$ Likewise, authors of the current study recommend future studies and clinicians consider use of mean reach (of the three trials) during perfor- 
mance analysis of YBT-LQ in this population, as this could reduce variation resulting in improved reliability.

Limitations of this study included variability of baseline activity levels among participants and allowing participants to wear preferred athletic shoes during the YBT-LQ. Both may limit direct comparison of the results of this present study to those of other YBT-LQ studies performed in adolescent populations. However, this study was intended for the outcomes to be more generalizable to the typical adolescent population, and did not control for any activities the subjects may have engaged in prior to testing sessions which may have affected a subject's performance during testing. It is also unknown how motivation could have an impact on subject performance, as this study was done on healthy adolescents who were not training for sport participation or recovering from injury. Attempts to reduce the effects of these limitations were made by testing subjects only one day apart at approximately the same time and by providing all subjects with consistent directions on both days of testing. Caution should be used when generalizing the results of this study to other populations or conditions. In addition, although the results of this study indicated mean reach to be a better measure of performance than maximum reach on the YBT-LQ in this population, the ANT reliability data was based on a smaller sample size due to participants being unable to complete this part of the test. Future studies are recommended on larger sample sizes, specifically to examine whether mean or maximum value of the ANT reach is optimal for this young adolescent population. Lastly, there was a significant difference between the number of boys and girls in this study. This discrepancy may limit the generalization of the results of the study, as the literature suggests differences in YBT-LQ performance between boys and girls. ${ }^{46-49}$ However, the gender factor was not the intended study variable of the study.

\section{CONCLUSION}

The results of this study suggest that leg dominance does not affect YBT-LQ performance in young non-athlete adolescents. In addition, the YBT-LQ, specifically the two posterior reaches, appears to be reliable for dynamic balance assessment in this population, whereas the composite score demonstrated poor reliability. Therefore, clinicians are advised to report the three reach scores separately. Use of the mean reach rather than maximum reach in each direction appears to have a better reliability for this population.

\section{CONFLICTS OF INTEREST}

The authors have nothing to disclose nor any conflicts of interest.

Submitted: June 14, 2021 CST, Accepted: October 13, 2021 CST 


\section{REFERENCES}

1. Geldhof E, Cardon G, De Bourdeaudhuij I, et al. Static and dynamic standing balance: test-retest reliability and reference values in 9 to 10 year old children. Eur J Pediatr. 2006;165(11):779-786. doi:1 $\underline{0.1007 / \mathrm{s} 00431-006-0173-5}$

2. Lee AJY, Lin WH. The influence of gender and somatotype on single-leg upright standing postural stability in children. J Appl Biomech. 2007;23(3):173-179. doi:10.1123/jab.23.3.173

3. Faigenbaum AD, Myer GD, Fernandez IP, et al. Feasibility and reliability of dynamic postural control measures in children in first through fifth grades. Int $J$ Sports Phys Ther. 2014;9(2):140.

4. Mickle KJ, Munro BJ, Steele JR. Gender and age affect balance performance in primary school-aged children. J Sci Med Sport. 2011;14(3):243-248. doi:1 $\underline{0.1016 / j . j s a m s .2010 .11 .002}$

5. Mohammadi V, Alizadeh M, Gaieni A. The effects of six weeks strength exercises on static and dynamic balance of young male athletes. Procedia - Soc Behav Sci. 2012;31:247-250. doi:10.1016/j.sbspro.2011.12.05 $\underline{0}$

6. Plisky PJ, Rauh MJ, Kaminski TW, Underwood FB. Star Excursion Balance Test as a predictor of lower extremity injury in high school basketball players. $J$ Orthop Sports Phys Ther. 2006;36(12):911-919. doi:1 $\underline{0.2519 / j o s p t .2006 .2244}$

7. Smith CA, Chimera NJ, Warren M. Association of Y Balance Test reach asymmetry and injury in division 1 athletes. Med Sci Sports Exerc. 2015;47(1):136-141. do $\mathrm{i}: 10.1249 / \mathrm{mss} .0000000000000380$

8. Wright AA, Dischiavi SL, Smoliga JM, Taylor JB, Hegedus EJ. Association of Lower Quarter Y-Balance Test with lower extremity injury in NCAA Division 1 athletes: An independent validation study. Physiotherapy. 2017;103(2):231-236. doi:10.1016/j.ph ysio.2016.06.002

9. Gonell AC, Romero JAP, Soler LM. Relationship between the Y Balance Test scores and soft tissue injury incidence in a soccer team. Int J Sports Phys Ther. 2015;10(7):955-966.

10. Hartley EM, Hoch MC, Boling MC. Y-Balance Test performance and BMI are associated with ankle sprain injury in collegiate male athletes. J Sci Med Sport. 2018;21(7):676-680. doi:10.1016/j.jsams.2017.1 $\underline{0.014}$
11. Plisky PJ, Gorman PP, Butler RJ, et al. The reliability of an instrumented device for measuring components of the Star Excursion Balance Test. North Am J Sports Phys Ther. 2009;4(2):92-99.

12. Garrison JC, Bothwell JM, Wolf G, et al. Y Balance TestTM anterior reach symmetry at three months is related to single leg functional performance at time of return to sports following anterior cruciate ligament reconstruction. Int J Sports Phys Ther. 2015;10(5):602-611.

13. Hallagin C, Garrison JC, Creed K, Bothwell JM, Goto S, Hannon J. The relationship between preoperative and twelve-week post-operative Y-Balance and quadriceps strength in athletes with an anterior cruciate ligament tear. Int J Sports Phys Ther. 2017;12(6):986-993. doi:10.26603/ijspt20170986

14. Kim K, Jeon K. Development of an efficient rehabilitation exercise program for functional recovery in chronic ankle instability. J Phys Ther Sci. 2016;28(5):1443-1447. doi:10.1589/jpts.28.1443

15. Kline PW, Johnson DL, Ireland ML, Noehren B. Clinical predictors of knee mechanics at return to sport after ACL reconstruction. Med Sci Sports Exerc. 2016;48(5):790-795. doi:10.1249/mss.0000000000000 $\underline{856}$

16. Hall EA, Docherty CL, Simon J, Kingma JJ, Klossner JC. Strength-training protocols to improve deficits in participants with chronic ankle instability: A randomized controlled trial. J Athl Train. 2015;50(1):36-44. doi:10.4085/1062-6050-49.3.71

17. Kang MH, Lee DK, Park KH, Oh JS. Association of ankle kinematics and performance on the Y-Balance Test with inclinometer measurements on the weightbearing-lunge test. J Sport Rehabil. 2015;24(1):62-67. doi:10.1123/jsr.2013-0117

18. Teyhen DS, Shaffer SW, Lorenson CL, et al. Clinical measures associated with dynamic balance and functional movement. J Strength Cond Res. 2014;28(5):1272-1283. doi:10.1519/jsc.000000000000 $\underline{0272}$

19. Brumitt J, Patterson C, Dudley R, Sorenson E, Hill G, Peterson C. Comparison of Lower Quarter YBalance Test scores for female collegiate volleyball players based on competition level, position, and starter status. Int J Sports Phys Ther. 2019;14(3):415-423. doi:10.26603/ijspt20190415 
20. Butler RJ, Bullock G, Arnold T, Plisky P, Queen R. Competition-level differences on the Lower Quarter Y-Balance Test in baseball players. J Athl Train. 2016;51(12):997-1002. doi:10.4085/1062-6050-51.1 $\underline{2.09}$

21. Butler RJ, Southers C, Gorman PP, Kiesel KB, Plisky PJ. Differences in soccer players' dynamic balance across levels of competition. J Athl Train. 2012;47(6):616-620. doi:10.4085/1062-6050-47.5.14

22. Gupta H, Yangdon T, Gupta U, et al. Normative data of dynamic balance of lower extremities using YBalance Test in cricketers with 16-25 years of age. IOSR J Sports Phys Educ. 2016;3(5):01-06.

23. Hannon J, Garrison JC, Conway J. Lower extremity balance is improved at time of return to throwing in baseball players after an ulnar collateral ligament reconstruction when compared to pre-operative measurements. Int J Sports Phys Ther. 2014;9(3):356-364.

24. Garrison JC, Arnold A, Macko MJ, Conway JE. Baseball players diagnosed with ulnar collateral ligament tears demonstrate decreased balance compared to healthy controls. J Orthop Sports Phys Ther. 2013;43(10):752-758. doi:10.2519/jospt.2013.46 $\underline{80}$

25. Hooper TL, James CR, Brismée JM, et al. Dynamic balance as measured by the Y-Balance Test is reduced in Individuals with low back pain: A cross-sectional comparative study. Phys Ther Sport. 2016;22:29-34. do i:10.1016/j.ptsp.2016.04.006

26. Shaffer SW, Teyhen DS, Lorenson CL, et al. YBalance Test: A reliability study involving multiple raters. Mil Med. 2013;178(11):1264-1270. doi:10.720 5/milmed-d-13-00222

27. Greenberg ET, Barle M, Glassmann E, Jung MK. Interrater and test-retest reliability of the Y Balance Test in healthy, early adolescent female athletes. Int J Sports Phys Ther. 2019;14(2):204-213. doi:10.26603/ii spt20190204

28. Schwiertz G, Brueckner D, Schedler S, Kiss R, Muehlbauer T. Performance and reliability of the Lower Quarter Y Balance Test in healthy adolescents from grade 6 to 11. Gait Posture. 2019;67:142-146. do i:10.1016/j.gaitpost.2018.10.011

29. Linek P, Sikora D, Wolny T, Saulicz E. Reliability and number of trials of Y Balance Test in adolescent athletes. Musculoskelet Sci Pract. 2017;31:72-75. doi:1 $\underline{\text { 0.1016/j.msksp.2017.03.011 }}$
30. Promsri A, Haid T, Werner I, Federolf P. Leg dominance effects on postural control when performing challenging balance exercises. Brain Sci. 2020;10(3):128. doi:10.3390/brainsci10030128

31. Brophy R, Silvers HJ, Gonzales T, Mandelbaum BR. Gender influences: the role of leg dominance in ACL injury among soccer players. Br J Sports Med. 2010;44(10):694-697. doi:10.1136/bjsm.2008.051243

32. Cug M, Ozdemir RA, Ak E. Influence of leg dominance on single-leg stance performance during dynamic conditions: An investigation into the validity of symmetry hypothesis for dynamic postural control in healthy individuals. Türkiye Fiz Tip Ve Rehabil Derg. 2014;60(1):22-26. doi:10.5152/tftrd.201 $\underline{4.59354}$

33. Hoffman M, Schrader J, Applegate T, et al. Unilateral postural control of the functionally dominant and nondominant extremities of healthy subjects. J Athl Train. 1998;33(4):319-322.

34. Alonso AC, Brech GC, Bourquin AM, Greve JMD. The influence of lower-limb dominance on postural balance. Sao Paulo Med J. 2011;129(6):410-413. doi:1 $\underline{0.1590 / \mathrm{s} 1516-31802011000600007}$

35. Promsri A, Haid T, Federolf P. How does lower limb dominance influence postural control movements during single leg stance? Hum Mov Sci. 2018;58:165-174. doi:10.1016/j.humov.2018.02.003

36. Mala L, Maly T, Zahalka F. Postural performance in the bipedal and unipedal stance of elite soccer players in different age categories. Acta Kinesiol. 2017;11:101-105.

37. Bigoni M, Turati M, Gandolla M, et al. Balance in young male soccer players: dominant versus nondominant leg. Sport Sci Health. 2017;13(2):253-258. d oi:10.1007/s11332-016-0319-4

38. Muehlbauer T, Schwiertz G, Brueckner D, Kiss R, Panzer S. Limb differences in unipedal balance performance in young male soccer players with different ages. Sports. 2019;7(1):20. doi:10.3390/sport $\underline{s 7010020}$

39. Breen EO, Howell DR, Stracciolini A, Dawkins C, Meehan WPI. Examination of age-related differences on clinical tests of postural stability. Sports Health. 2016;8(3):244-249. doi:10.1177/1941738116633437

40. Dhali B, Saha GC. Comparison of dynamic balance between two legs of three growth stages of children. Int J Phys Educ Sports Health. 2016;3(6):385-388. 
41. Bulow A, Anderson JE, Leiter JR, MacDonald PB, Peeler J. The Modified Star Excursion Balance and YBalance Test results differ when assessing physically active healthy adolescent females. Int J Sports Phys Ther. 2019;14(2):192-203. doi:10.26603/ijspt2019019 2

42. Baxter-Jones ADG, Eisenmann JC, Sherar LB. Controlling for maturation in pediatric exercise science. Pediatr Exerc Sci. 2005;17(1):18. doi:10.1123/ pes.17.1.18

43. Faul F, Erdfelder E, Buchner A, Lang AG. Statistical power analyses using $G^{*}$ Power 3.1: tests for correlation and regression analyses. Behav Res Methods. 2009;41(4):1149-1160. doi:10.3758/brm.4 $\underline{1.4 .1149}$

44. van Melick N, Meddeler BM, Hoogeboom TJ, Nijhuis-van der Sanden MWG, van Cingel REH. How to determine leg dominance: The agreement between self-reported and observed performance in healthy adults. Macaluso A, ed. PLOS ONE. 2017;12(12):e0189876. doi:10.1371/journal.pone.018 $\underline{9876}$
45. Koo TK, Li MY. A guideline of selecting and reporting intraclass correlation coefficients for reliability research. J Chiropr Med.

2016;15(2):155-163. doi:10.1016/j.jcm.2016.02.012

46. Chimera NJ, Smith CA, Warren M. Injury history, sex, and performance on the functional movement screen and Y balance test. J Athl Train. 2015;50(5):475-485. doi:10.4085/1062-6050-49.6.02

47. Gorman PP, Butler RJ, Rauh MJ, et al. Differences in dynamic balance scores in one sport versus multiple sport high school athletes. Int J Sports Phys Ther. 2012;7(2):148-153.

48. Gribble PA, Robinson RH, Hertel J, Denegar CR. The effects of gender and fatigue on dynamic postural control. J Sport Rehabil. 2009;18(2):240-257. doi:10.11 23/jsr.18.2.240

49. Kramer TA, Sacko RS, Pfeifer CE, Gatens DR, Goins JM, Stodden DF. The association between the Functional Movement ScreenTM, Y-Balance Test, and physical performance tests in male and female high school athletes. Int J Sports Phys Ther.

2019;14(6):911-919. doi:10.26603/ijspt20190911 